\title{
LEGIBILITY
}

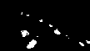

A major purpose of the Technical Information Center is to provide the broadest dissemination possible of information contained in DOE's Research and Development Reports to business, industry, the acadernic community, and federal, state and local governments.

Although a small portion of this report is not reproducible, it is being made available to expedite the availability of information on the research discussed herein. 


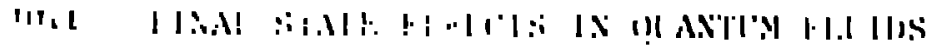

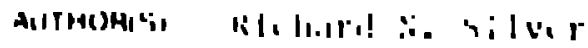

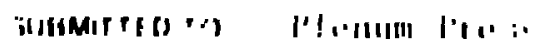

\section{IDW I.AIMF.K}

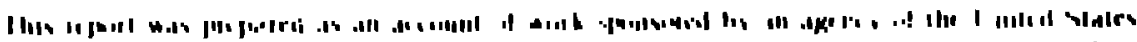

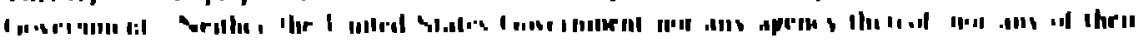

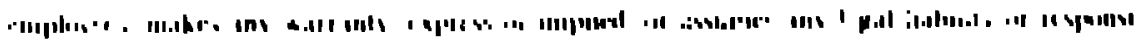

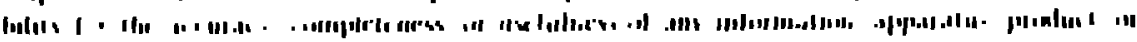

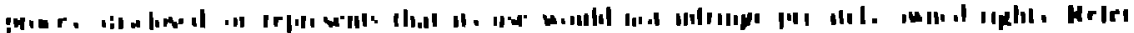

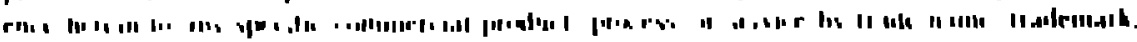

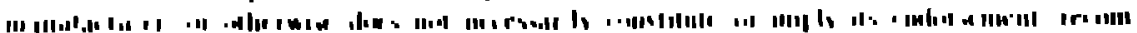

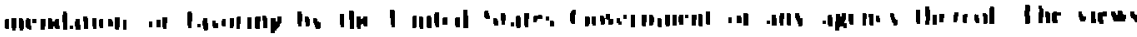

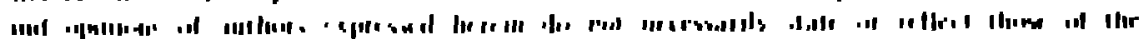

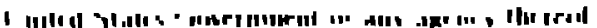

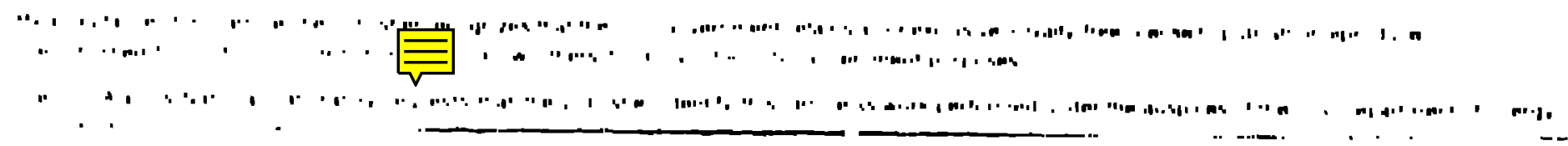

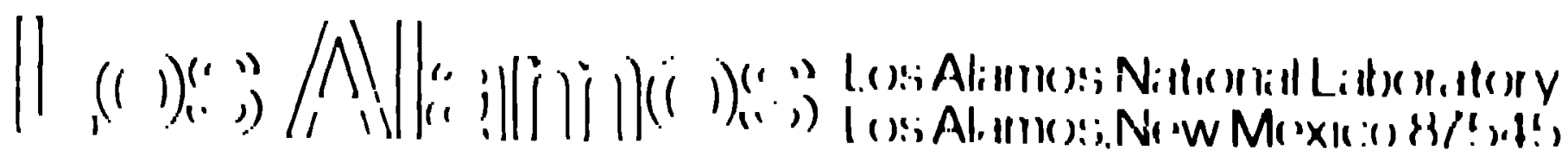
ind $1 ., \ldots$

i i. I. 
FINAL STATE EFFECTS IN QUANTUM FLUIDS

\author{
R'ichurd I. Silver \\ Theoretical Division and I os Alamos Neutron Scattering Center \\ MS B 202 las Alamos National L aboratory \\ lons Alamus, New Mexico 87545
}

\title{
INTRODUCTION
}

The extraction of momentum distributions from high energy scattering experiments depends on the validity of the impinlse approximation (IA), which has heere extensively discusied in the wortshop overview $|1|$. In the IA

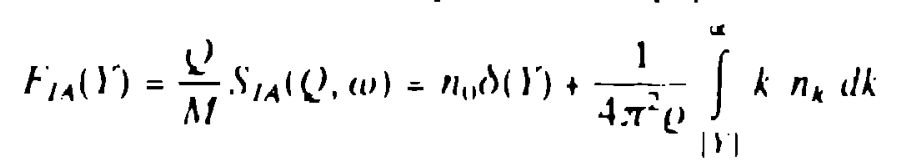

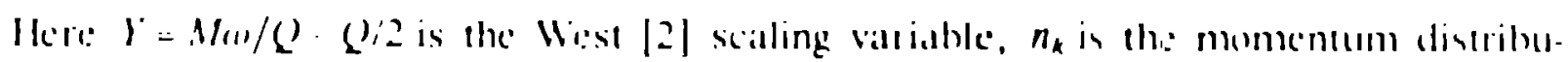
tion, $n_{0}$ is the bese comdensatte fraction in the case of superfluid 4 flic, and $e$ is the density. The IA asssumes that in the scattering process the hencetic encergy imparted to

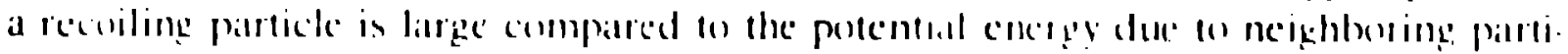

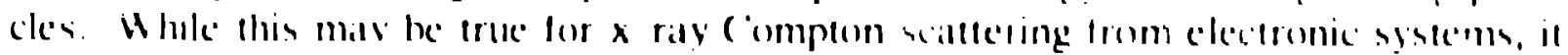

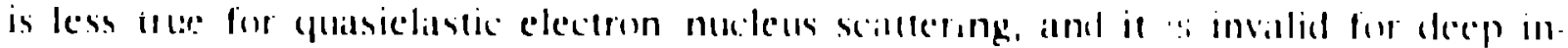

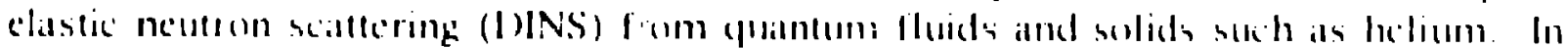

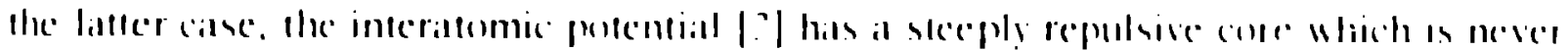

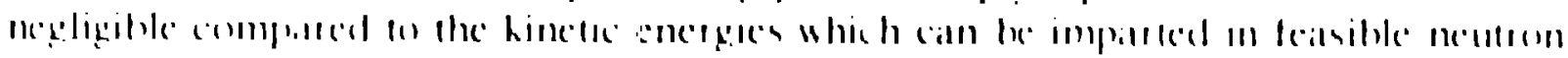

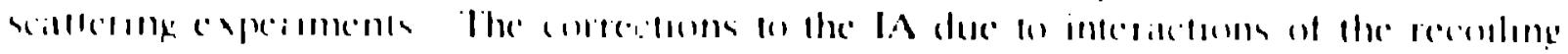

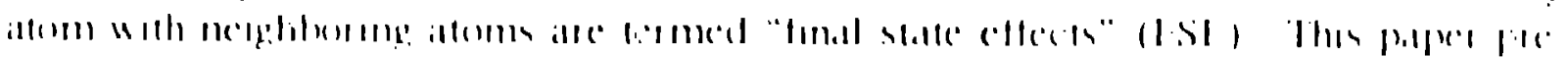

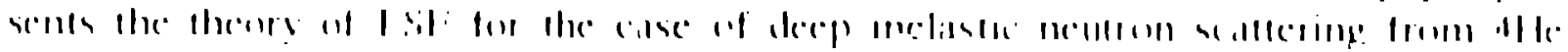

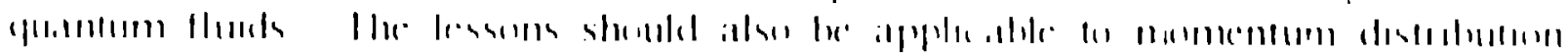

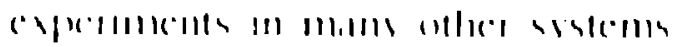

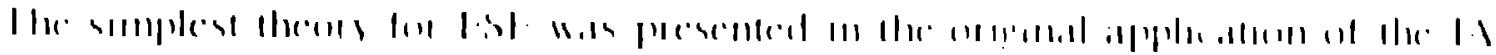

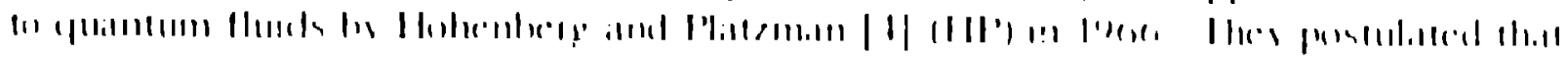

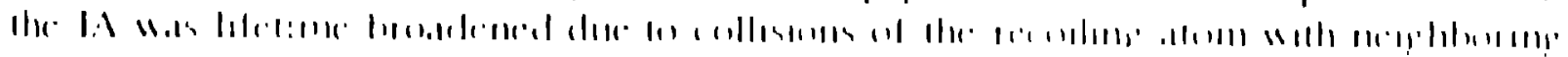

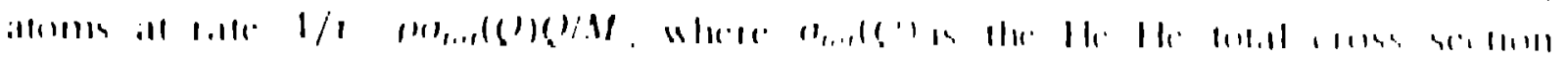

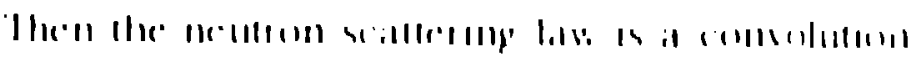




$$
F(Y) \equiv \frac{Q}{M} S(Q, w)=\int_{x} d \gamma^{\prime \prime} R\left(Y-Y^{\prime \prime} \mid F_{H, A}()^{\prime \prime}\right) .
$$

where the broadening predicled by III is lorentzian

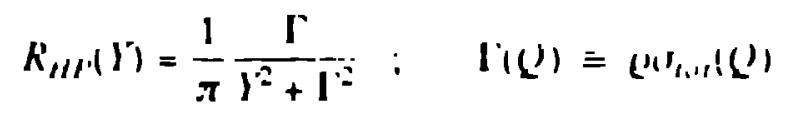

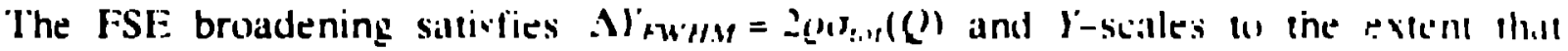

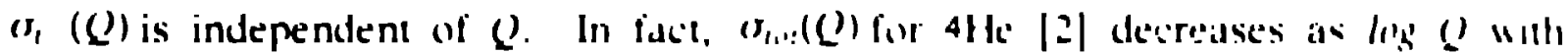

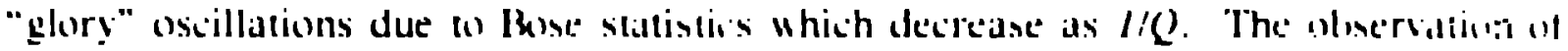

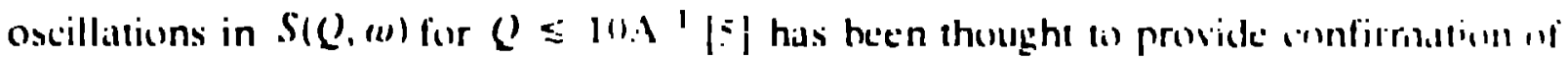
the $1 \mathrm{ll}$ theory. However, the $\omega^{2}$-suin rule on $S(Q,(U)$ repuires

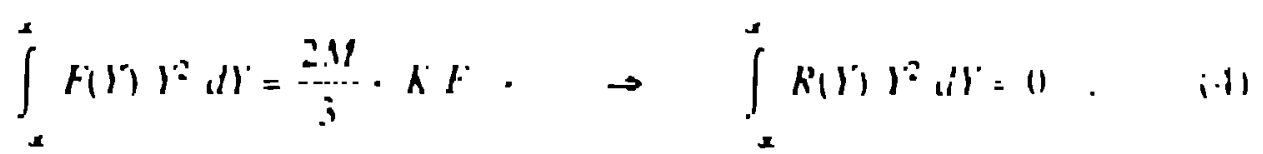

which is violated by the I oremuian tails predicted by the III' the

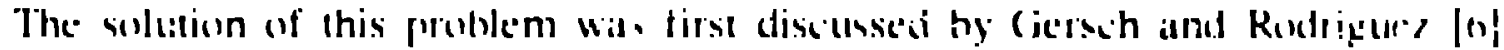

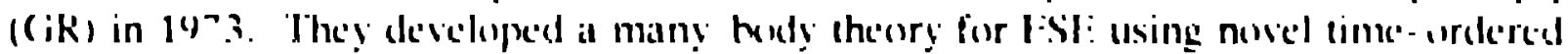

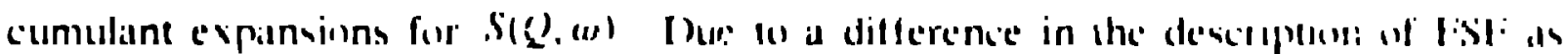
dephasing rather than lifetime trobadening, $R(Y)$ at small $\left|l^{\prime}\right|$ should he positive hul

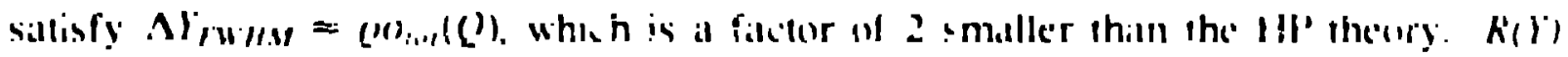

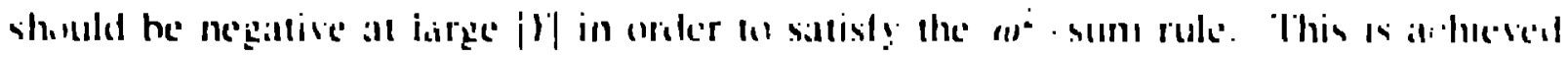

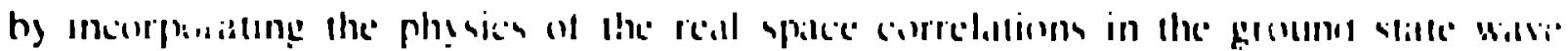

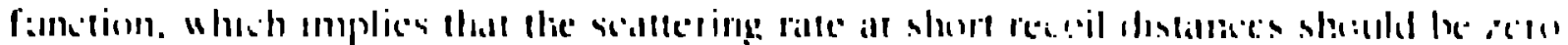

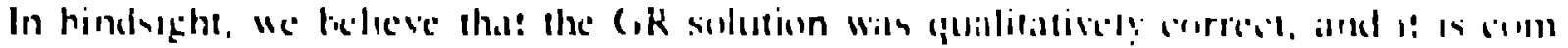

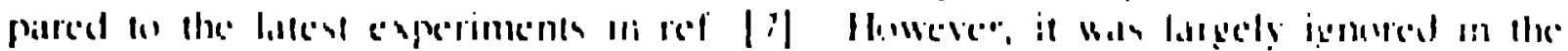

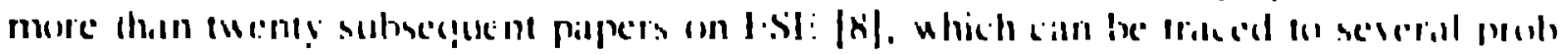

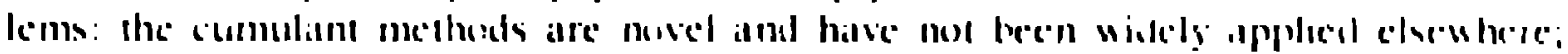

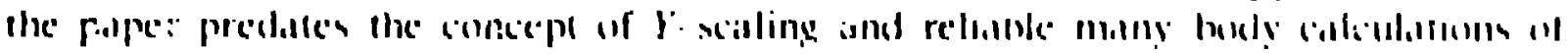

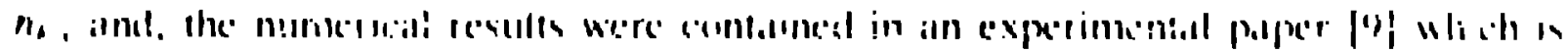

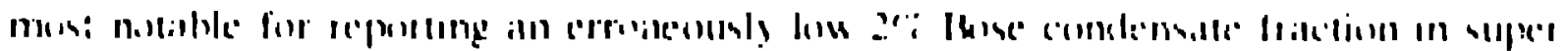
Ilinis:

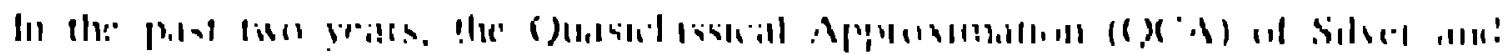

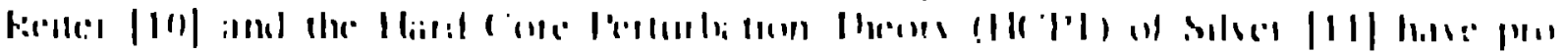

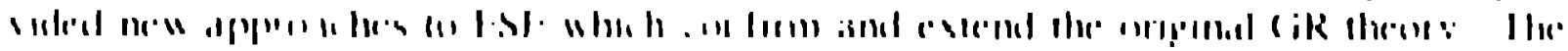

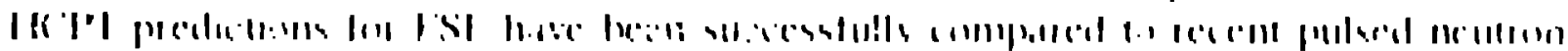

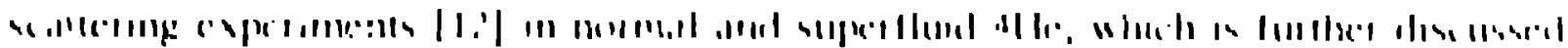
by simms.

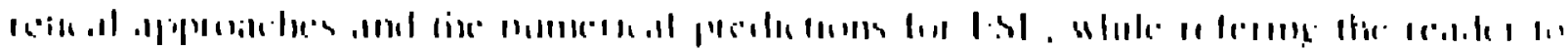

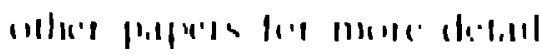

\section{OUASICI.ASGICAL APPROXIMATIDN}

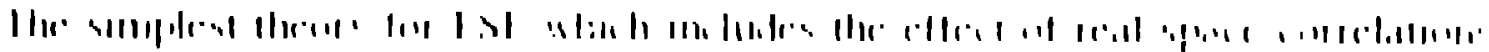

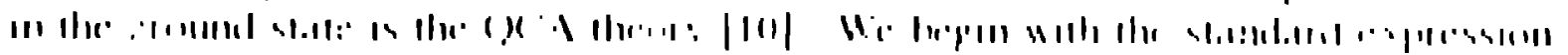

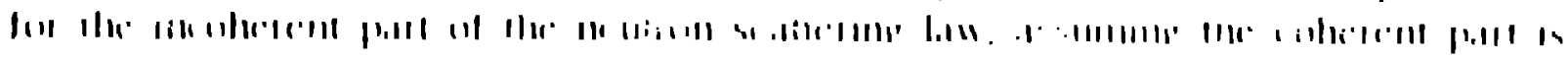




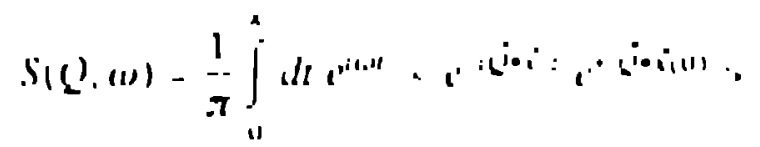

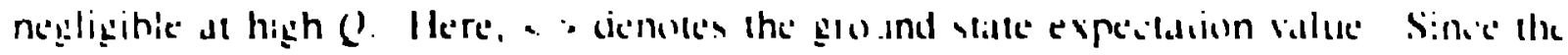

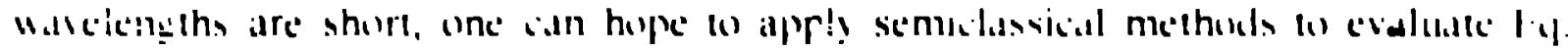

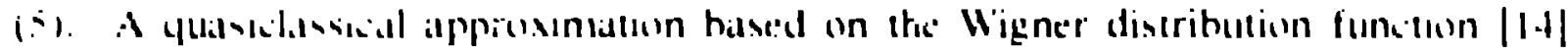

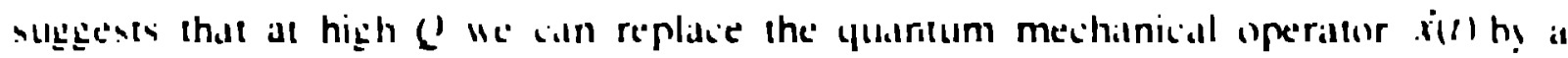

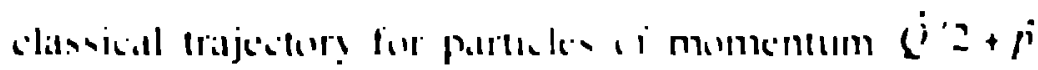

$$
\left.(1)-\frac{(i t}{211}+\frac{p_{1}}{11}+\operatorname{din}\right)
$$

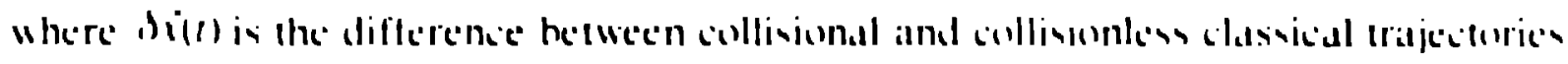
The'n

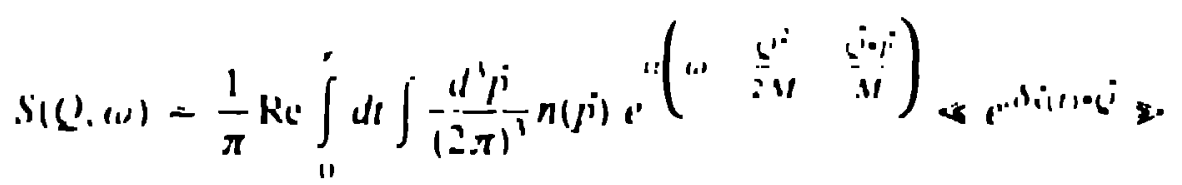

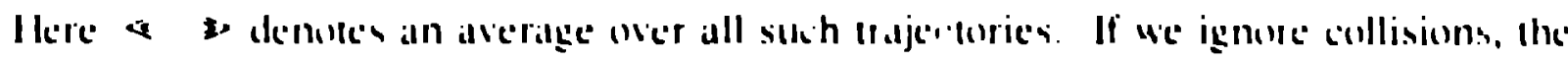

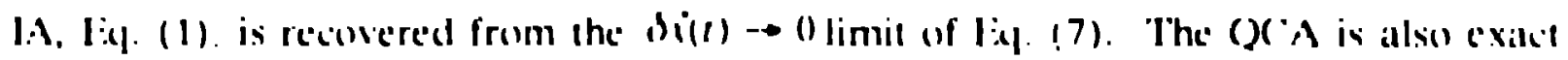

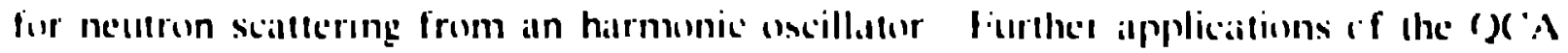
are dincilseed li! Reiller $|1.5|$

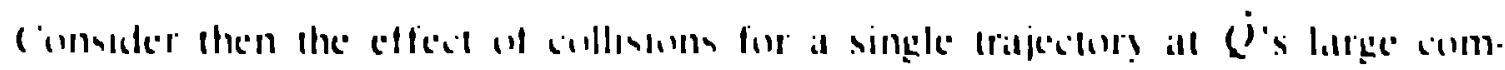

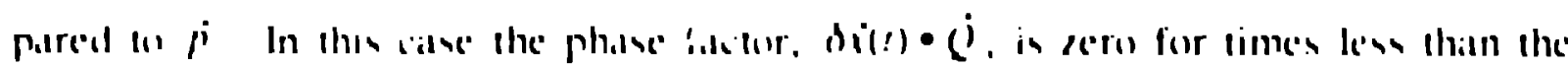

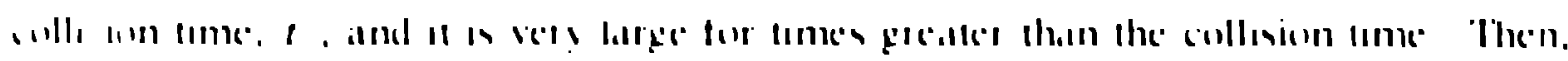

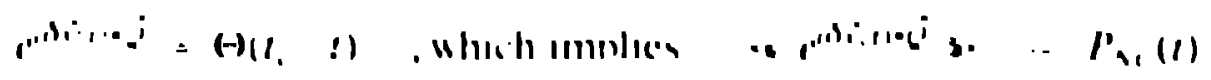

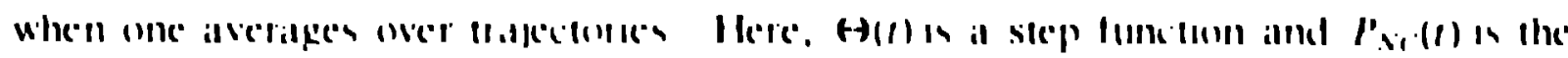

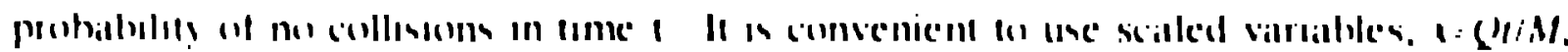

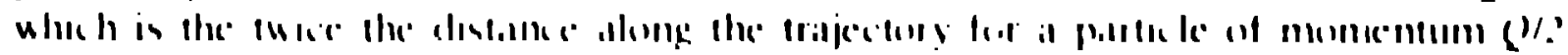

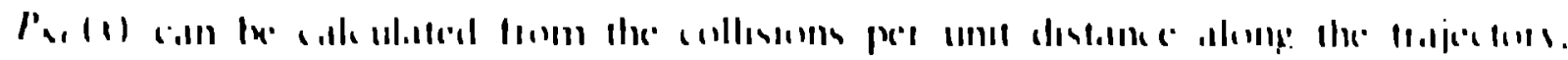

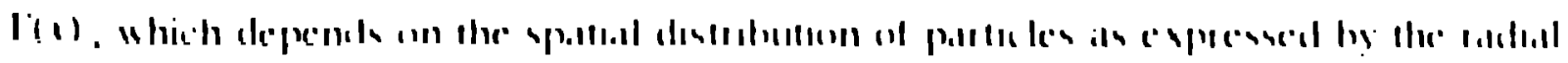

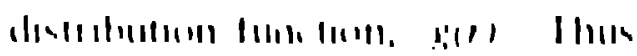

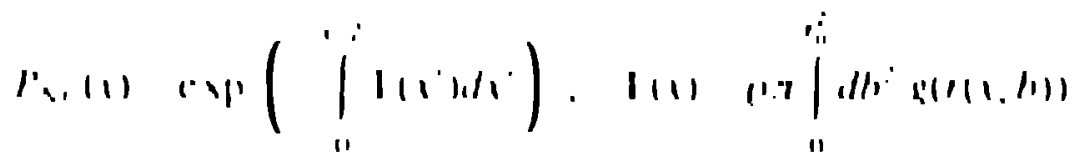

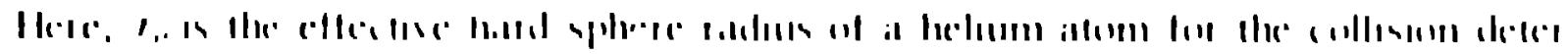

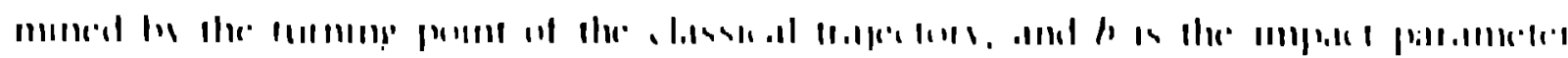

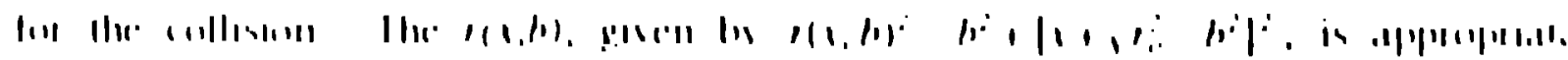

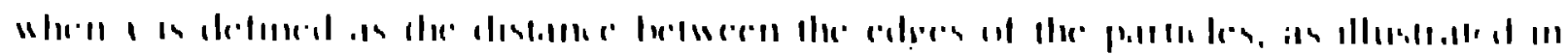

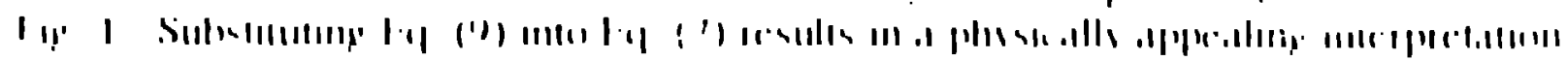

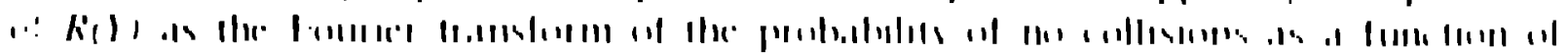

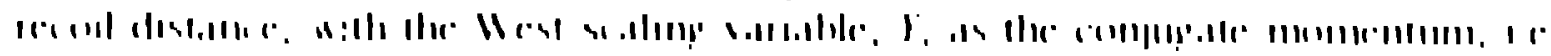




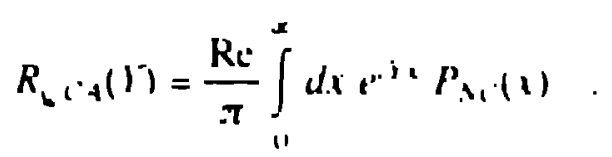

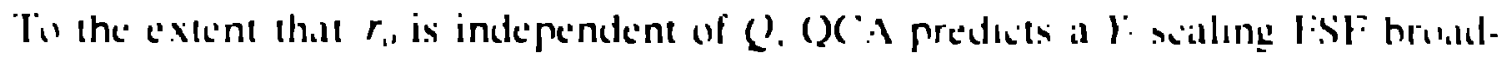
ening In the limil of ignoring reall space correlations, i.e. g'tr) $\rightarrow$ I, the III' the'o!,

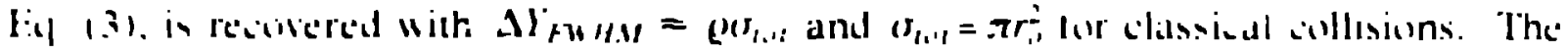
inclusion of real space correidtions hals al dramatic elfect. als illustrulted in lige. 2 .

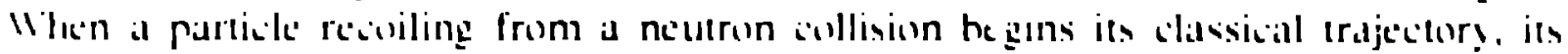

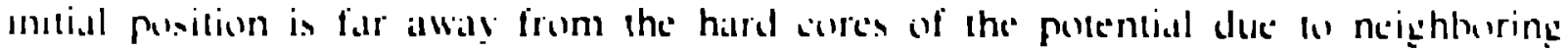
aloms which are ésponsible for final state effects. It must trast some distanic nefore il encounters a hard core, so there are no collosions all small r. Thus P, $(1)$ is

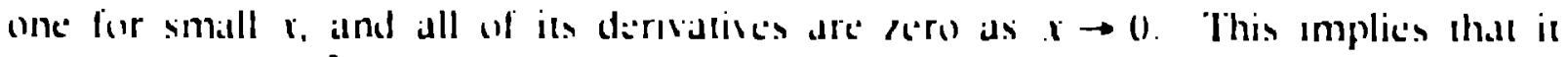

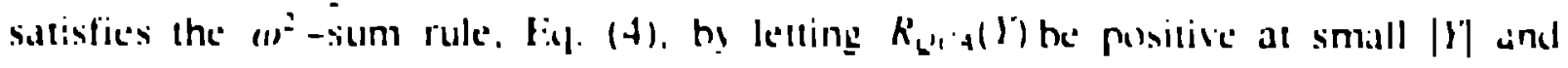

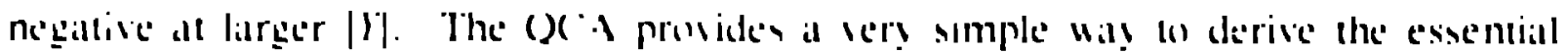
yualiuative phssics of l:s!:

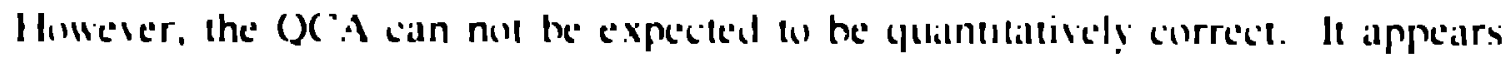
to te plagued hy a number of lackors at 2 . (Jne difficuly can be traced to the neglest

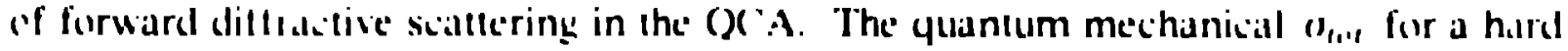

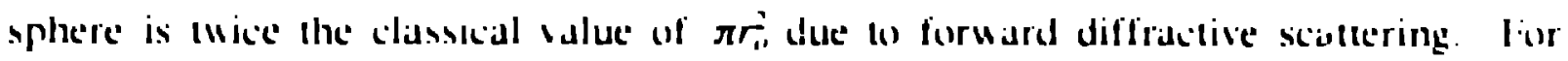

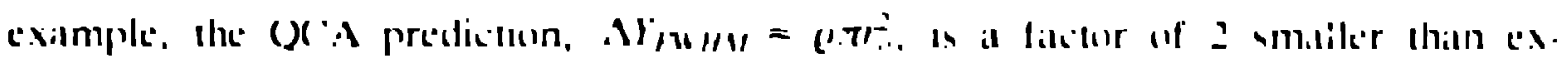

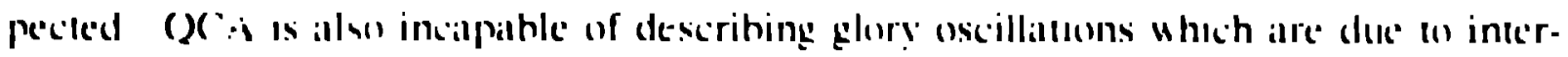

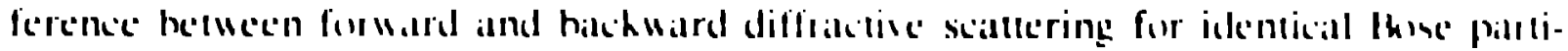

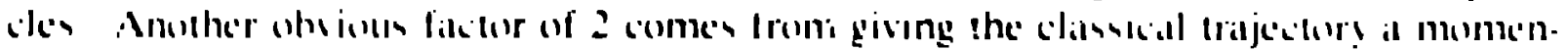

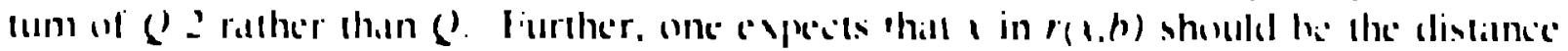

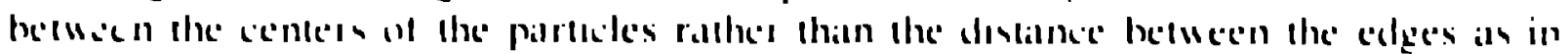

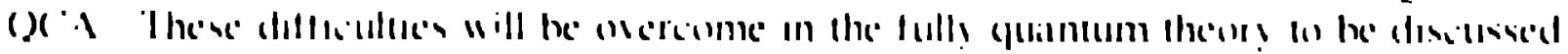

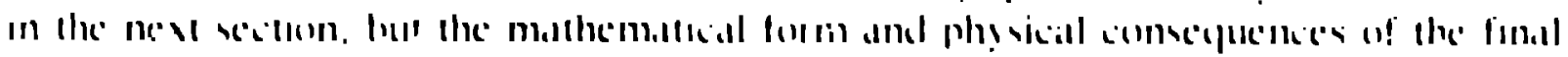

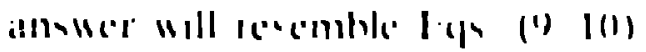

\section{THE QUANTUM THEORY OF FSE}

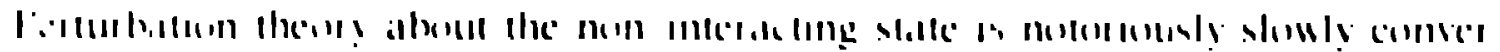

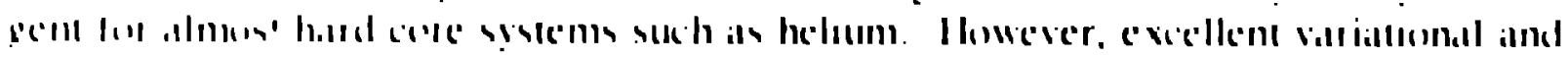

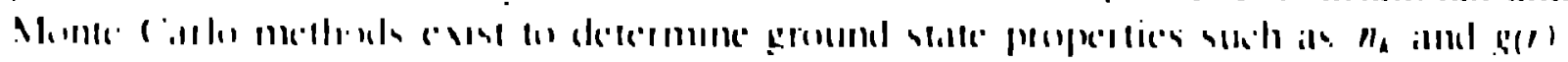

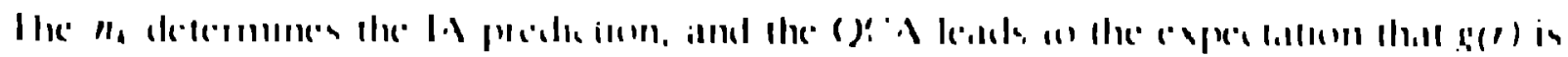

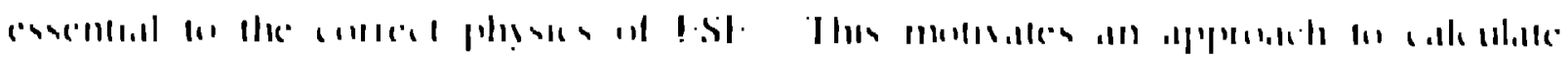

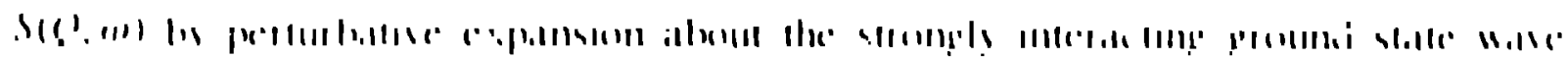

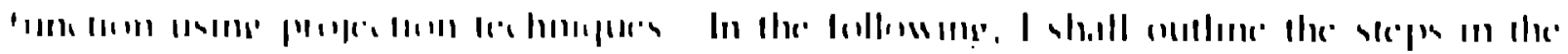

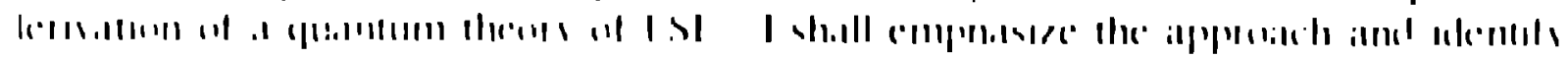

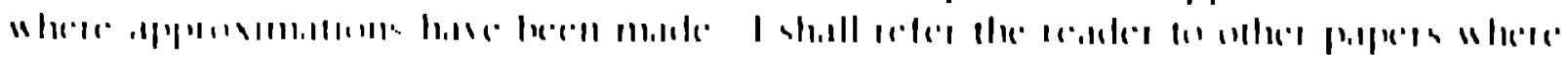

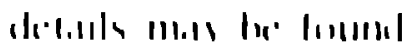

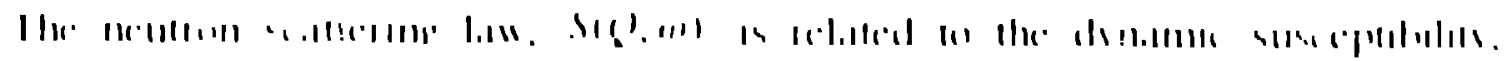
(1!!, (11), 1

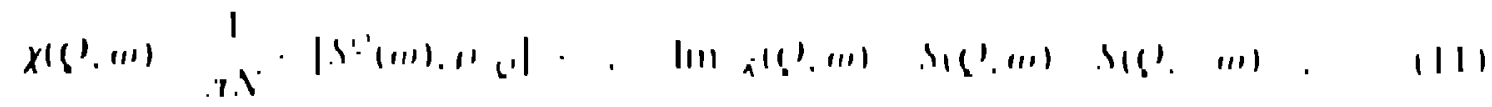


whure

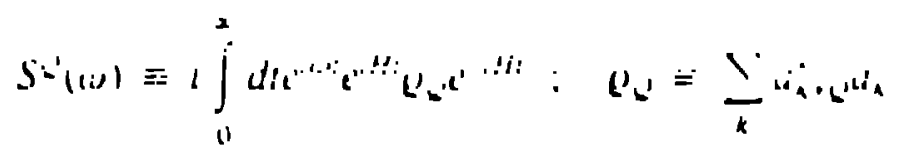

This heth component af the dy namic suseeptibilits, $\chi_{k}$. mat be deloned by

$$
x_{a} \equiv \frac{1}{\pi .1} \cdot \mid S^{\prime}(u), u_{a}^{*},\left(a_{n} \mid>\right.
$$

The'n diagianmatic perturhation theory can prowe that the $\chi_{A}$ satisfy an eadet

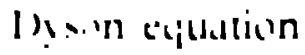

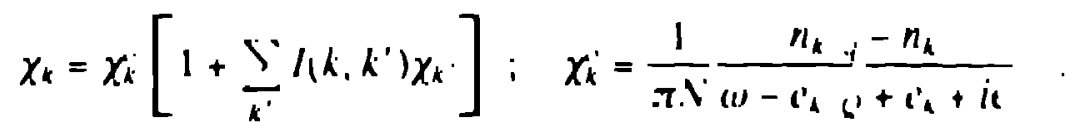

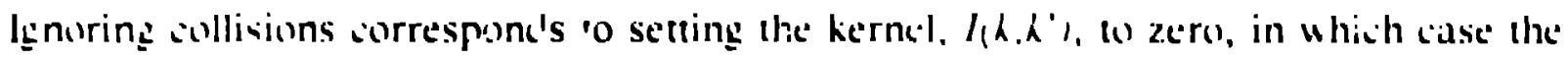
IA is recosered from F.y. (14). ihe kernel is calculated $|11,16|$ h: truncating a projecetion superoperator expansion of $\chi(Q)$. (1)) at lowest order in the two-hody t--matrix and keeping unly the non-zero ternss in the "asymptotic limit" of high $Q$ and hard core interalitions.

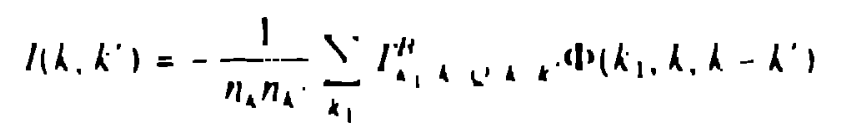

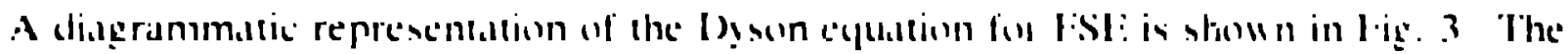

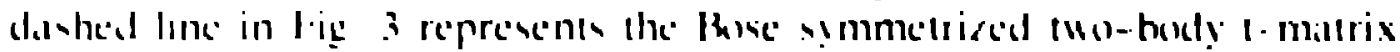

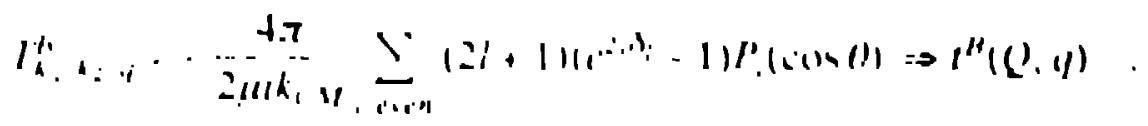

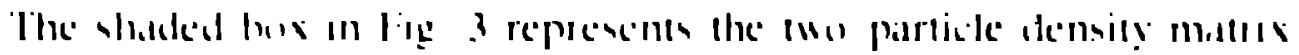

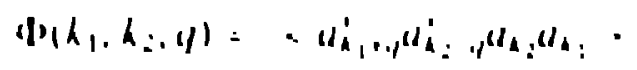

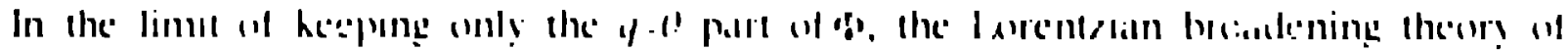

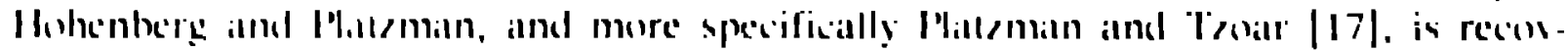

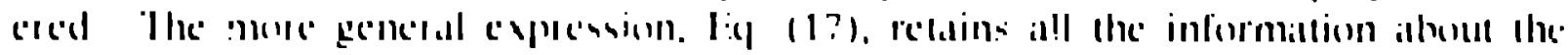

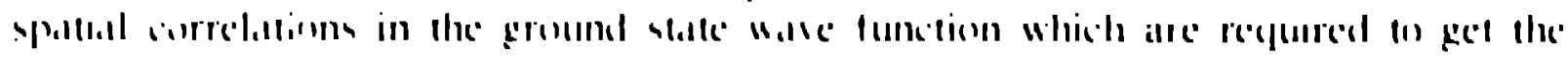

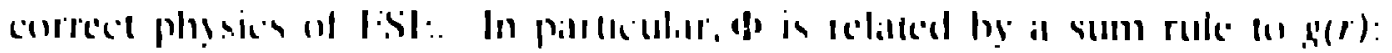

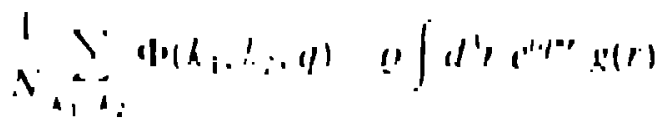

$\therefore M$,

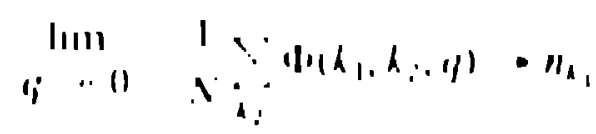

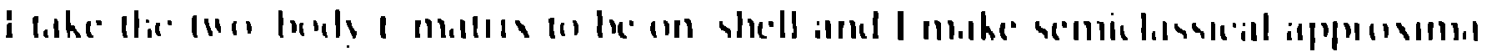
IIIm)

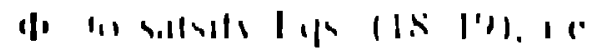

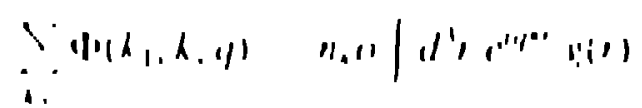

L |

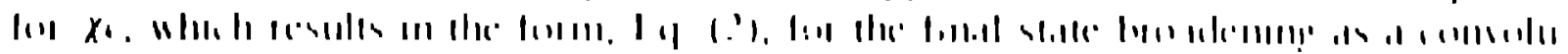

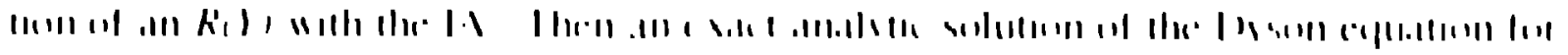




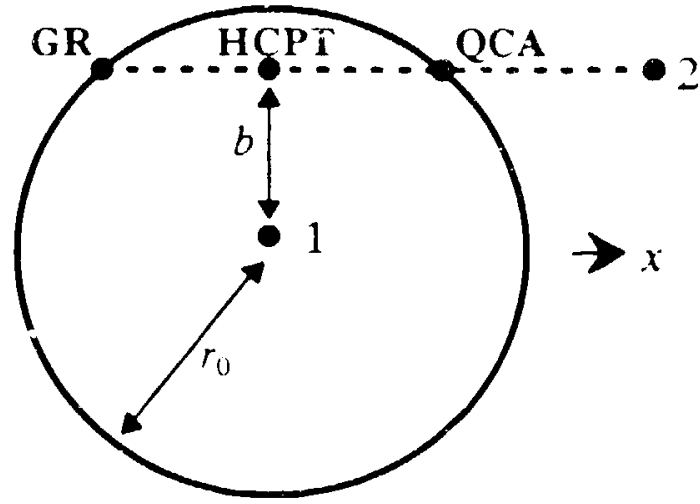

CLASSICAL TRAJECTORIES

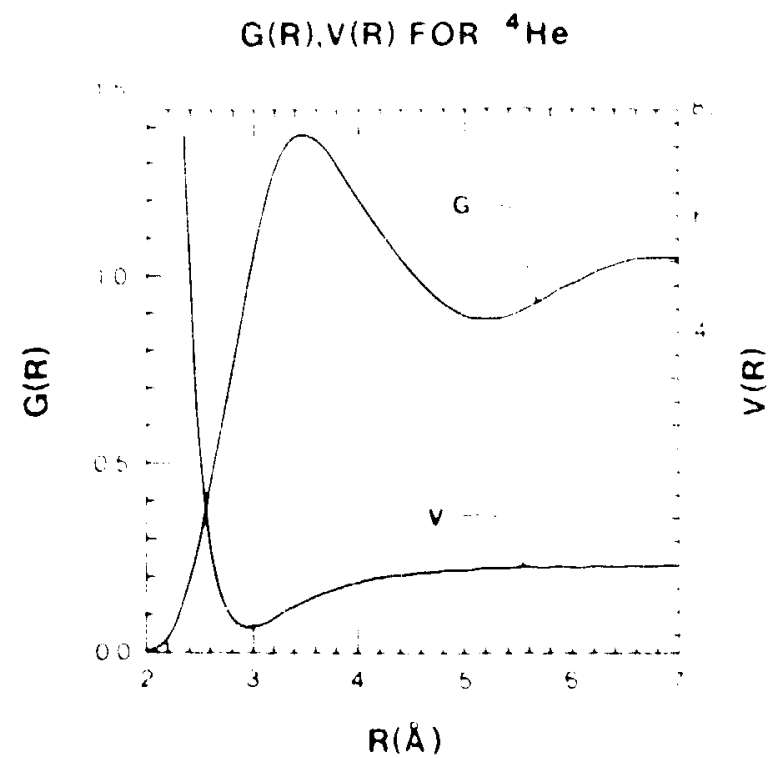

F!G. 2 - Plot of the radial distribution functlon, $g(r)$. for $4 \mathrm{He}$ and the $\mathrm{He}-\mathrm{H}_{\mathrm{e}}$ potential, $V(r)$. After neutron collision, the recoiling particle starts Its trajectory in the attractive well of the potential at some distance from the steeply repulsive core responsible for $f i$ nal state affects. The scaling variable, $Y$, is conjugate momentum to distance, $x$, siong the classical trajectory.

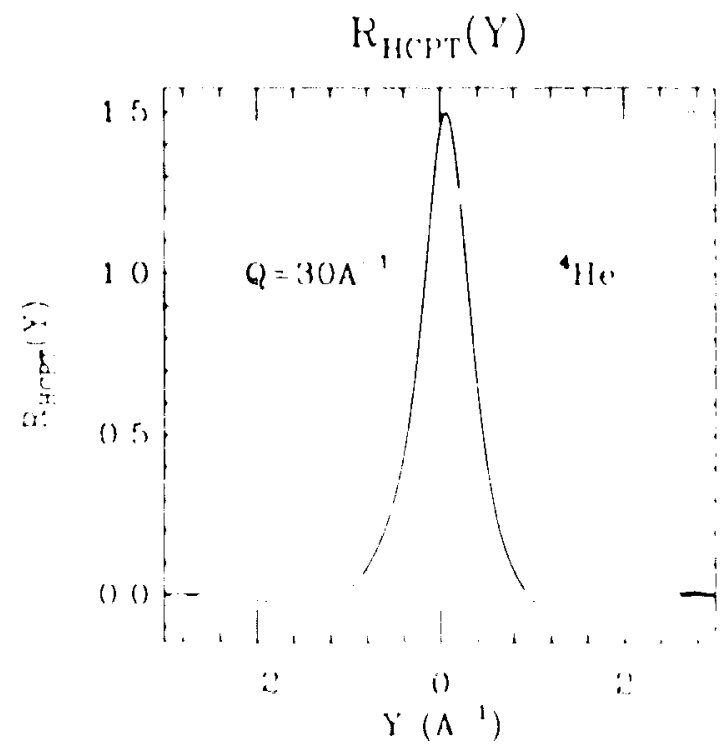

FIG. 4 - The final state offects (FSE) broad. ening function, Hil, predicled by HCPT for 4He. 
the $\chi_{k}$ exists, details of which may he found in ref. [11].

The final result for $R\left(Y^{\prime}\right)$ is identical to $\mathrm{El}$. (10) obtained by the QCA, with

$$
P_{x} \cdot(x)=\exp \left(-\int_{0}^{x} \Gamma\left(x^{\prime}\right) d x^{\prime}\right) ; \quad I(1)=0 . \pi \int_{0}^{x} d h^{2} f_{b} g(r(x, b)) .
$$

This is similar to Eq. (9) but differs in three details. First, there is no factor of 2 in the limit of the integral. Second,

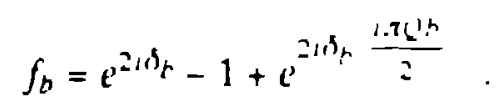

Here, $\delta_{b}$ is the semiciassical (JWKB) phase shift for He-He scattering at impact parameter $b$. The third term in Eq. (22) is due to Bose symmetrization and gives rise to the hard sphere glories in the He-He total cross section. The real part of $\Gamma(1)$ is again the collisions per unit distance. W'ith $\operatorname{Re} \Gamma(x)=\varrho \sigma_{t o r} / 2$ by the optical theorim, there is an interesting cancellation of the 2 in $\sigma_{t, t}=2 \pi r_{v}$, so that $\operatorname{Re} \Gamma(x)$ is simply the classical result, $\varrho \pi r_{\nu}^{2}$. Finally, $r(x, b)=\sqrt{x^{2}+b^{2}}$ for HCPT. The $x$ 's appropriate for the HCPT, QCA and GR theorics are shown in Fig. 1.

\section{HCPT PREDICTIONS FOR EXPERIMENT}

The HCPY predictions [19] for deep inelastic neutron scattering on $4 \mathrm{He}$ can be evaluated using as input the variational and Monte Carlo $n_{k}$, the experimental $g(r)$ measured hy neutron diffraction [20|, and the semiclassical predictions or the He-He phase shifts. The semiclassical methods are accurale at high l. especially since the He-le potential at short distances is inferred from measured $\sigma_{t, t}$ by invertung the semiclassical formulae |3|.

Figure + shows the $R\left(l^{\prime}\right)$ predicted at $Q=30 \wedge{ }^{\prime}$ which is achiciable al pulsed neutron sources, but is much larger than reactor experiments for which $Q \leq 12 \mathrm{~A}$.

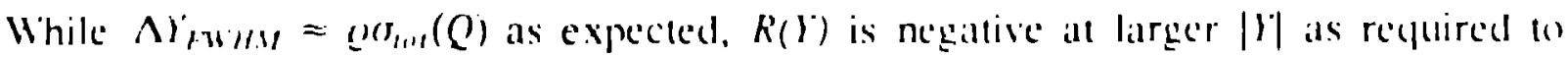
satisfy the $w^{2}$-sum rule. Figure 5 shows specilically how this is accomplished by displaying the integrand of the sum rule, $l 2 k\left(l^{\prime}\right)$. The sum rule reguires the ared under this curve to be zero. The sciale of the oscillations in $l 2 R(l)$ is determined by

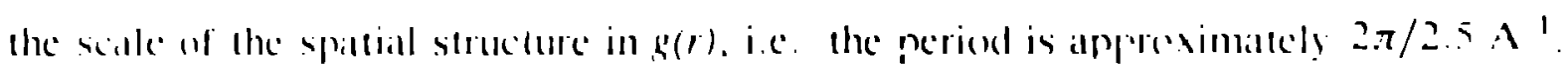

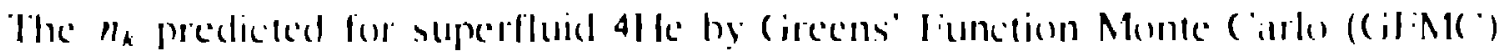

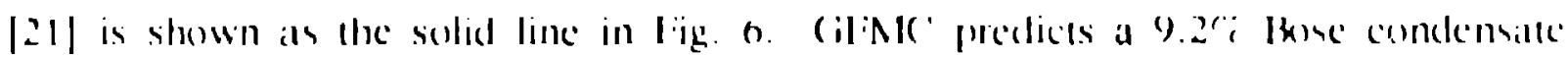

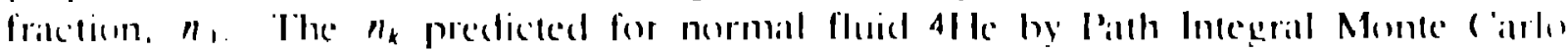

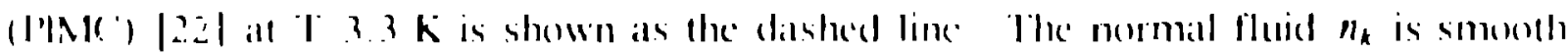
and appormamiltely (iamssian

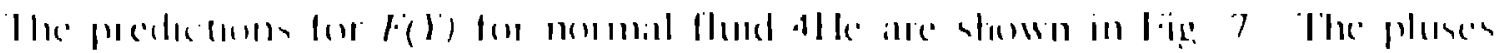

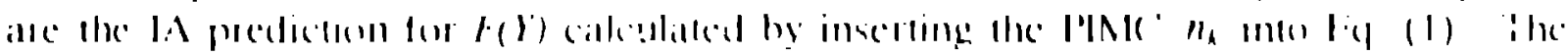

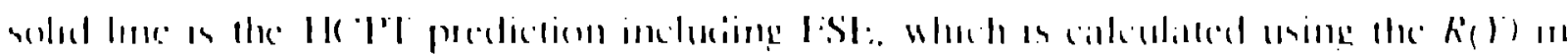

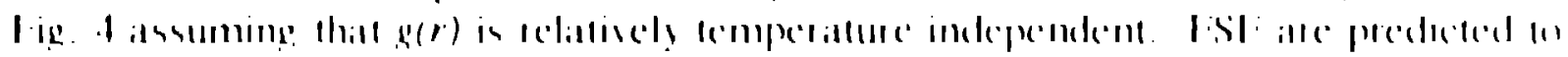




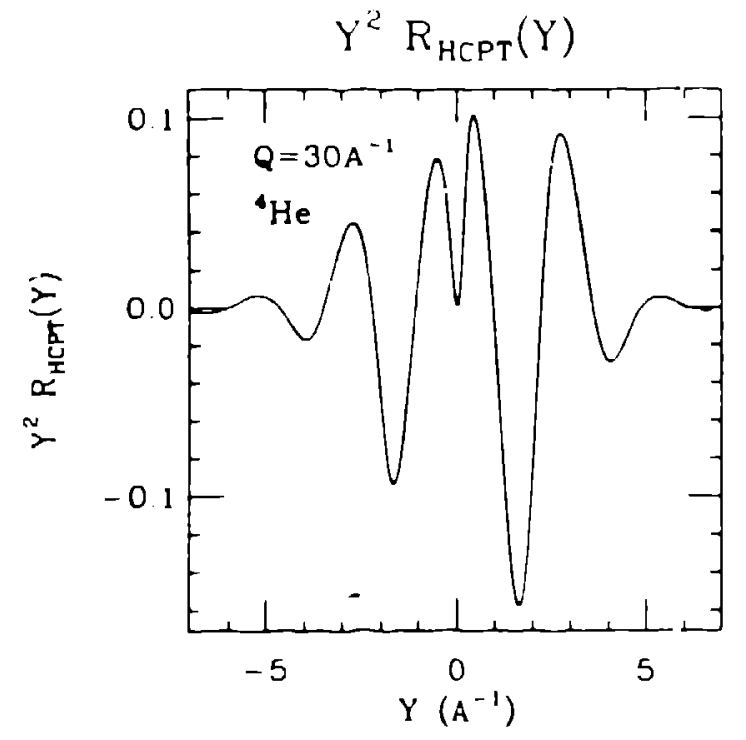

FIG. 5 - $Y^{2} R(Y)$ predicted by HCPT. The $\omega^{2}$ eum rule requires the erea under thle curve to be zero. The ecale of the etructure is governed by Fourler transform of the radial distribution function, $g(r)$.

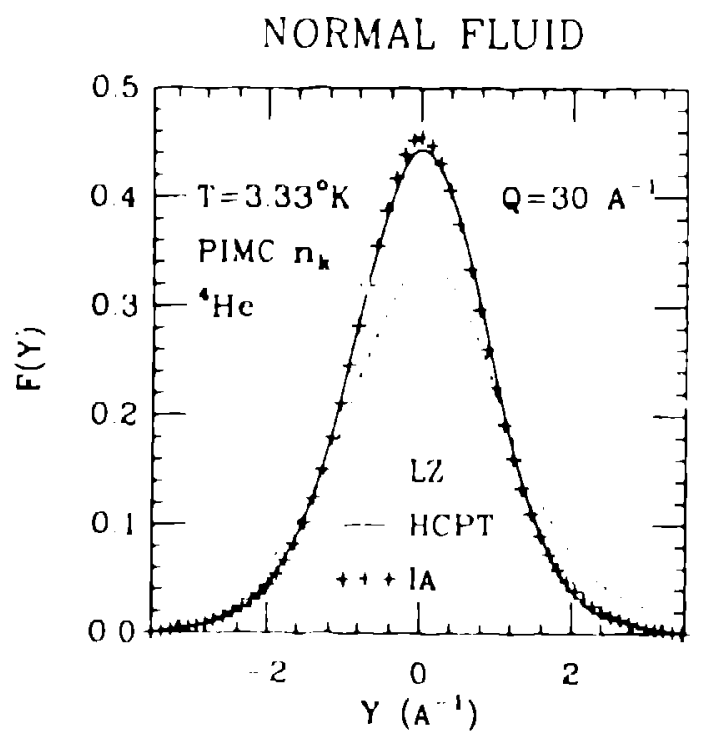

FIG. 7 - The neutron ecaltering lew, $F(Y)$, for normal "He of $\mathrm{T}=3.33 \mathrm{~K}$ using the PIMC momentum distribution. The pluses are the impulse approximation (IA) prediction. The solid line te the HCPT prediction which includes broadening due to final state effects (FSE). The dashed line is the Lorentzian (LZ) broadening prediclion oblained by $\mathrm{lg}$ noring ground state spatial correlatione and cetting $g(r)$ to one.

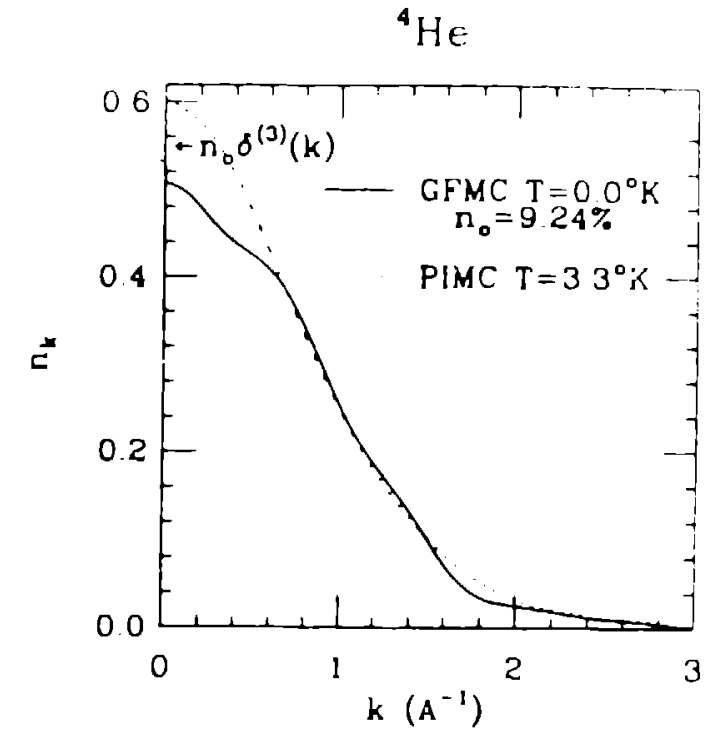

FIG. 6 - Momentum distributions for liquld ‘He. The eolid line ic the Greene' Function Monte Carlo (GFMC) predlction for the superfluld at $T=O K$, which has a delta function at $k=0$ with $9.2 \%$ Bose condensate fracIion. The dashed line is the Path integral Monte Carlo (PIMC) prediction for the normal fluid at $T=3.33 K$.

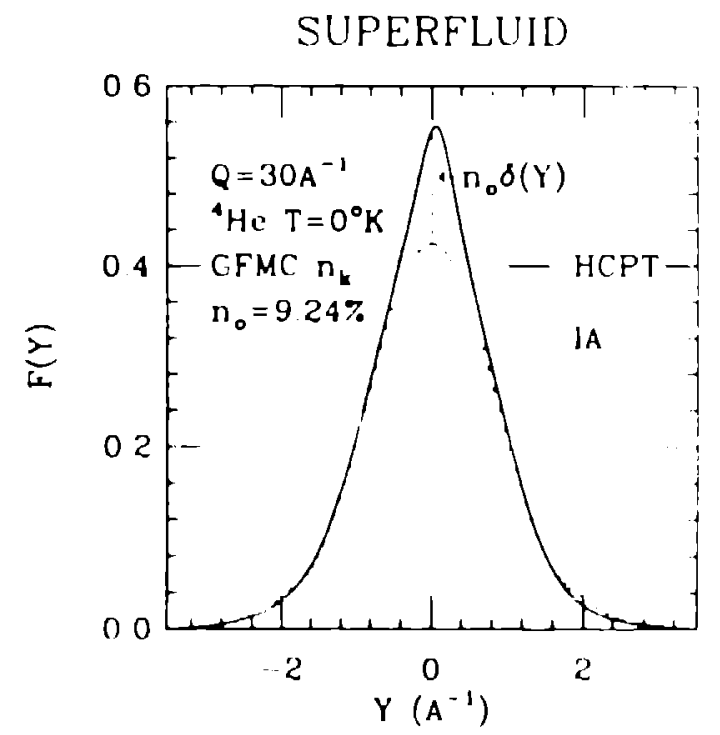

FiG. 8 - The neutron ecattering law, $F(Y)$, for superfluid "He at $T=O K$ using the GFMC momentum distribution. The dashed line is the Impulse approximation (IA) prediction. which has a delta function at $Y=0$ due to the Bose condensate with $9.2 \%$ of the weight. The solid line is the prediction of HCPT which includes broadening due to linal state alfects (FSE). 
be almost negligible for the normal fluid at $\Omega=30 \mathrm{~A}^{-1}$, and the LA should be an excellent description. For comparison, the dashed line is the prediction of the Lorentzian broadening theory obtained by setting $g(r)$ to one. The FSE predicted by HCPT are much smaller than those predicted by theories which ignore spatial correlations. This is expected since the effect of spatial correlations is to reduce cotlisions at short recoil distances.

The corresponding IA and HCPT predictions of $F\left(Y^{\prime}\right)$ for the superfluid are shown in Fig. 8. The IA is calculated by inserting the IJFMC $n_{k}$ in Fig. 6 into Eq. (1). The dashed line is the IA prediction which has $9.2 \mathrm{c}$ of the weight in a delta function at $Y^{\prime}=0$. FSE are included in the HCPT prediction shown by the solid line, which is calculated by convoluting the IA prediction with the $R\left(Y^{\prime}\right)$ in Fig. 4, according to Eq. (2). The Bose condensate de!ta function in the LA is completely wiped out by FSE broadening, so that it is no longer a distinct peak. However, the Gaussian width of the overall distribution is unchanged by FSE as is axpected from the $\omega^{2}-s u m$ rule. Eq. ( 4 ). Intensity is robbed from high $\left|Y^{\prime}\right|$ in order to preserve the sum rule.

Thus, the Bose condensate peak will not become resolvable by going to $Q$ 's much larger than reactor experiments. However, such $Q$ 's permit a theory of FSE, which allows tests of calculations of $n_{k}$ using DINS datci. This prediction does not imply that values of $n_{0}$ can not be inferred directly from DIS.IS data, but the problem has become ill-conditioned as is discussed by Sivia and Silver [23] in these proceedings.

Since this theory of FSE predicts that the Bose condensate is unobservable as a distinct peak at $Q=30 \mathrm{~A}^{-1}$, a critical question becomes at what $Q$ 's the Bose condensate may become observable as a distinct peak. As in the original HP theory, the broadening scales with $\sigma_{t w r}(Q)$ for $4 \mathrm{He}$, which is very slowly decreasing with increasIng $Q$. Figure 9 shows the classical turning point, $r_{0}$, of the He-Hle potential [3] as a function of $Q$ on a linear-log scale, which shows that the decrease is approximately' logarithmic. Figure 10 shows the slow approach to the IA predicted by HCPT by plotting $F\left(l^{\prime}\right)$ at several $Q$. HCPT FSE vary as $\left.O_{i} Q^{0}\right)$ for hard core potentials, and approximately as $O(\log ())$ for the He-He potential. Experimentalists should certuinly try to test this prediction by experiments at much larger $Q$. However, $Q$ 's greater than $100 \mathrm{~A}{ }^{\prime}$ with adecuate resolution are unfeasible with present spectrometers.

The hehavior of ISSE should be similar for IINS experiments on 3lle fiermi licquid, where a primary goal is to observe the Fermi surface discontinuity predicted in $n_{k}$. The solid line in Yigg. 11 is the Fermi HyperNetled Chain (I:HNC) prediction |24] for $n_{k}$ which hass a discontinuity. and the dashed line is the prediction of the 1 hullier and Bouchaud wave function [2.5| which does not. The lSSE predicted by HCYY anil the $f(l)$ all $Q=3() \wedge$ ' for 3 le using the FINC $n_{k}$ as input have been calculated. An apparent $n_{k}$ could be inferred from a measured $f(Y)$ by alsisuming the IA was corred

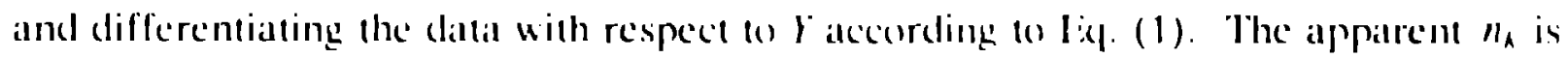

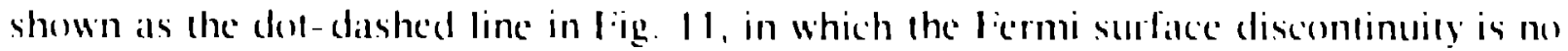

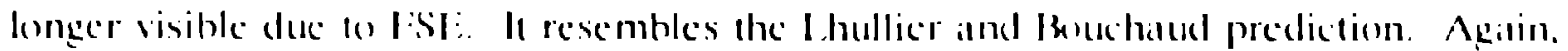

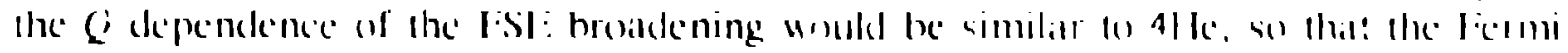

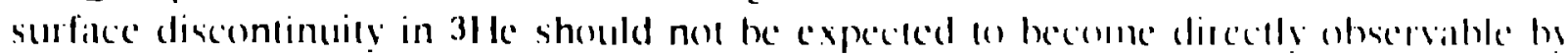
performing experiments all much higher (') 


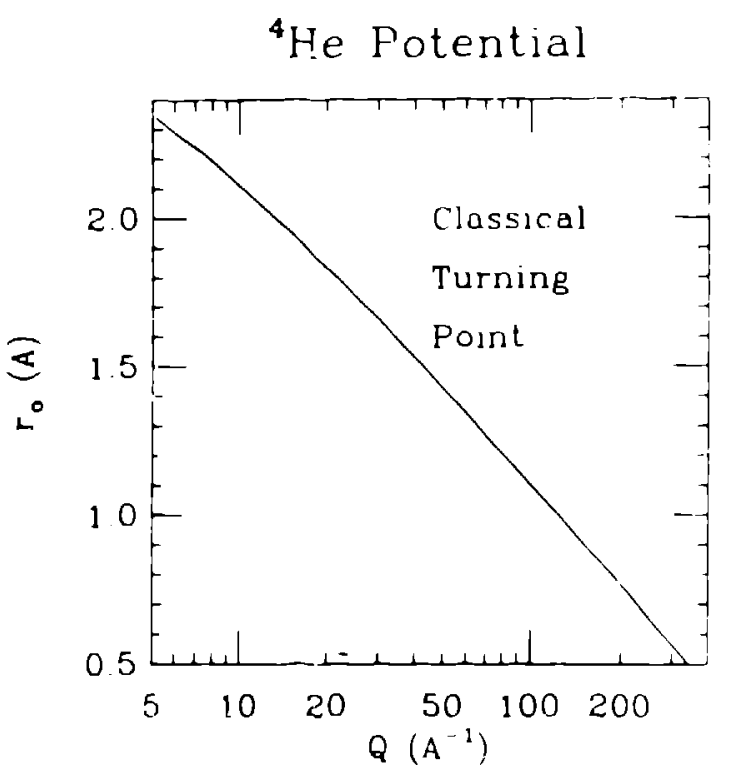

FIG. 9 - Classical turning points of the HeHa potential versus $Q$. plotted on linear-log scale. This shows that the width of the FSE broadening of the IA, $R(Y)$, should decrease epproximatoly as $O(\log Q)$ with increasing $Q$.

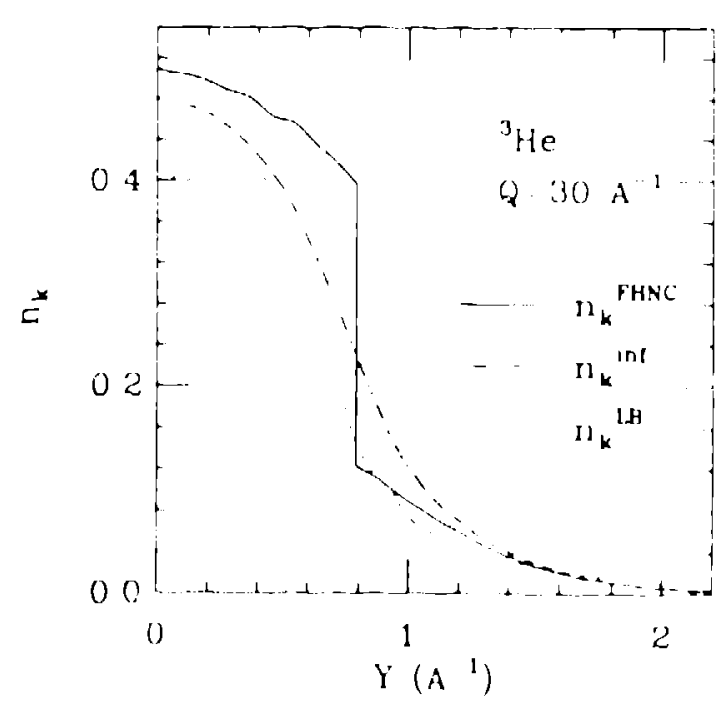

FIG. 11 - Momentum distributlons for normal JHe Ferml liquid at T=OK. The eolid line lo the Forml Hypernotted Chain (FHNC) prediclion. The dashed lins is the prediction using the Lhullier Bouchaud (LB) wave function. The dot-dash line to the apparent momentum distrlbution after broadening the FHNC-IA neutron scatlering law by the HCPT predicliaii inr FSE. The Fermi curface disconfinulty is omooinet out by FSE.
Approach to the IA

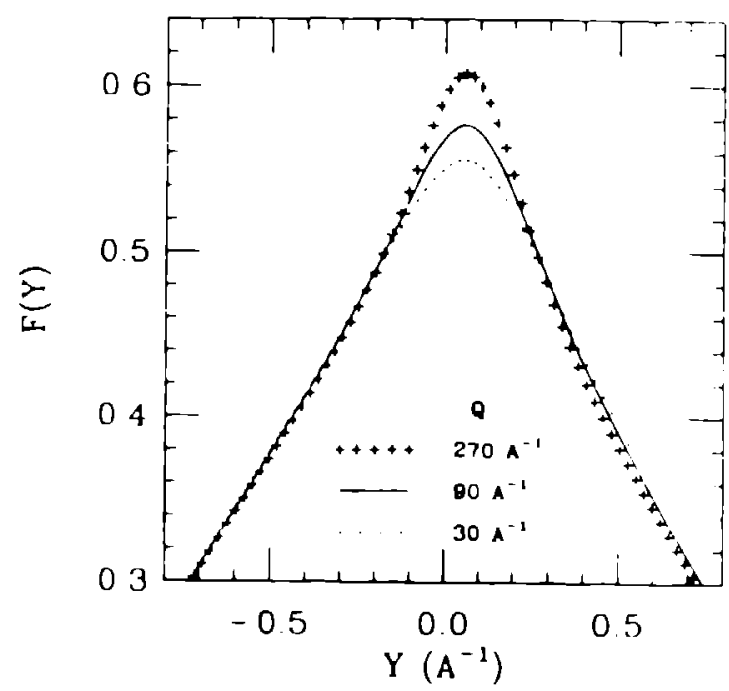

FIG. 10 - Approach ts the impulse approximation (IA) with Increasing $Q$ of the neutron ccattering law, $F(Y)$, for euperfluid "He at $T=O K$ as predicted by HCPT. Only the region near $Y=0$ relevant to the Bose condensate is shown. The Bose condensate does not become distinct peak at any experimentally feasible 0 .

\section{Glory Oscillations}

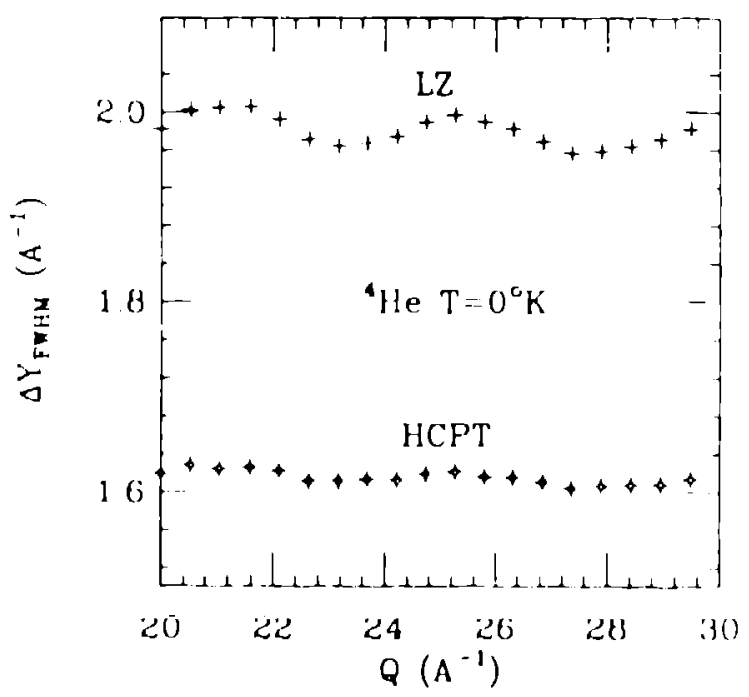

FIG. 12 - Full Width Half Maximum (FWHP of the neutron scattering law, $F(Y)$, for $s$. perfluld " $\mathrm{He}$ at TxOK including FSE brondenIng. The HCPT to at ihe boltom. The Lorentzlan broadening prediction (LZ) at the top le obtained by setting $g(r)$ to one. The FWHM and the glory osclllations are much emaller for HCPT than for $L Z$. They are close to the Impulse approximaiton (IA) prediction. 
The direct comparison of the HCPT predictions for $F\left(I^{\prime}\right)$ with pulsed neutron experiments at $\mathcal{Q}=23 \mathrm{~A}^{-1}$ on $4 \mathrm{He}$ is discussed in rei. [12], and in the paper by Sosnick, et al. [13] in these proceedings. The agreement is excellent with no adjustible parameters. A discussion of the formal relarion of HCIT to other theories maly he found in ref. [8], and a comparisun between the daia and these theories is presented in ref. [7j. HCPT has the best agreement with experiment. Given this success, one may question the need for improvements. However, in addition to further experimental tests and applications to other sy'stems, there remain many outstanding problems in the theory of FSE.

First, 'e approximation of the two-paricle density matrix ( (1) by Eq. (20) satısfies Eqs. (18-19), but it does not satisfy other known properties of d. Recently Ristig ind Clark [26] have calculited a general structure for (1) which remedies this defect. By substitutiag their expression for $\Phi$ into Eṇs (14-15), orie can formally show that the convolution form of the FSE, Eq. (2), and the relative temperalure independence of FSF should be regarded as a'pproximations. The critical issue remaining is whether the excellent agreement between theory and experiment [12| becomes fortuitous after the corrections to the IA using better $\Phi$ are calculated.

The apryroximations of IICPT, such as the semiclassical treatment oi the t-matrix, begnin to faii at the lower $Q$ 's of reactor experiments. W'hile IICPl has kept the

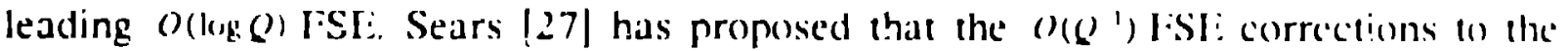
lA are approximately antisymmetric in $Y$. They can he the dominam corrections in cases where the HCIY ISSI: are small, eg. the almost Gaussian rinmentum distributions such as for normal 4 tte as shown in fig. 7. There appears such a slight asymmetric component even in the $Q=2.3 \mathrm{~A}^{-1}$ experiments $|12|$, and such an asymmetry is pronounced in reactor experiments on 4 lle and other noble gases. The glory ascillit-

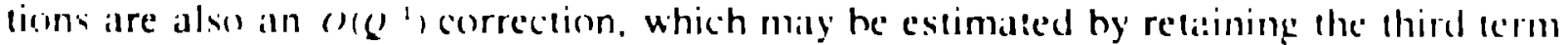
in 1 (y 122). I:igure 12 shows thate the glory oscillations should be moth smaller than predicted hy the Iarentian hrobdening theories which ignore ground state spattial correliations. Ilowever, a complete theory for FSI: which includes all the 110 ' 1 lerms

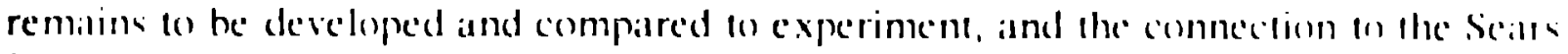
[27] expansion of ISSI: in powers of $(1$ needs to be clarified.

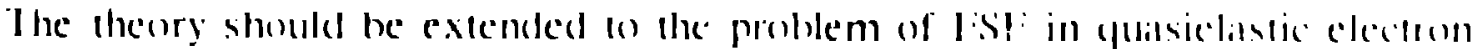

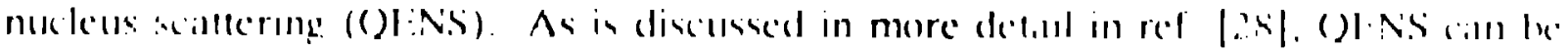

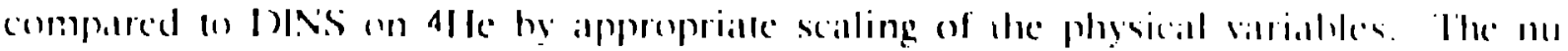

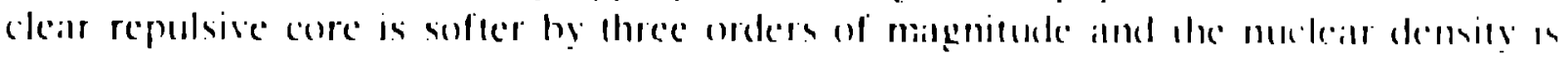

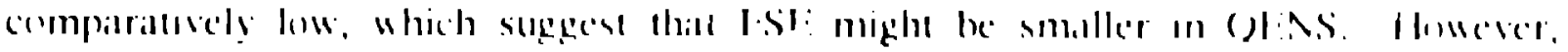

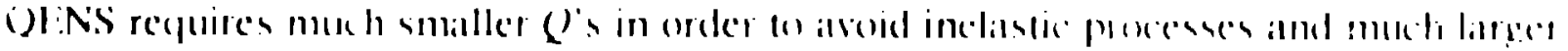

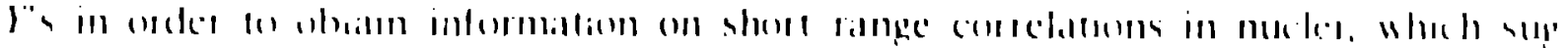

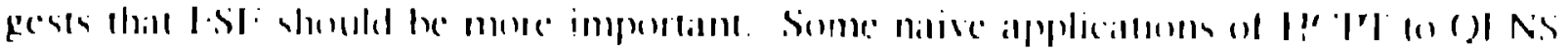

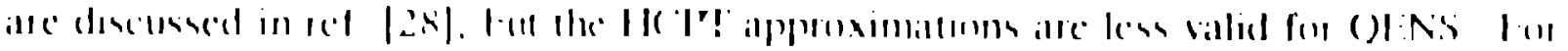

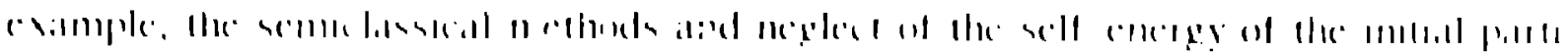

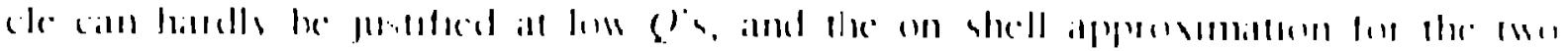

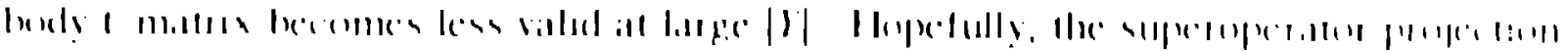

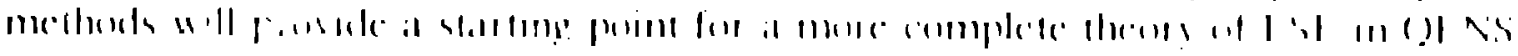




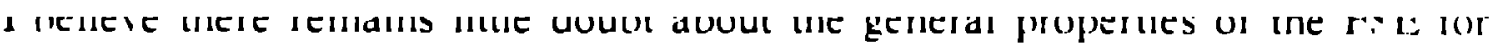
DINS in helium. However, the quasiclassical approximation and the supersperator expansions 1 have used in their derivation are no more familiar to the condensed matter physics community than the time-ordered cumulant expan ions originally used by Gersch and Rodriguez $[6]$. All of these methods deserve further development, as well as applications to other problenss involving the dynamical resporise of systems with streng ground state corrs: ttions. In addition, a treatment of FSE using diagrammatic perturbation theory starting with the non-interacting ground state remains 10 be provided.

\section{ACKNOWLEDGEMENT}

Research supported by the Office of Basic Fenergy Sciences of the U.S. Dept of Energy. I thank K. Bedell, J. W. Clark, G. Rieter, and P. L. Sokol for many helpful discussions.

\section{REFERENCES}

[1] J. W'. Clark, R. N. Silver, P. E. Sokol, this volume.

[2] G. IB. W'est, I'hysics Reports 18. 263 (1975).

[3] R. Feltgen, H. Kirst, K. A. Koehler, I: Torello, J. Chem. Ihy's. 26, 2360 (1982).

[4] P. C. llohenberg, P. M. Platzman, Phys. Rev. 152. 198 (1966).

15) P'. Niankil, I: (. Sienssun, A. I) B. Woods, V. I: Sears, R. A. Couley, J. I aw Temp. Phys. 23, 28.5 (1976).

[6] II. A. (jersch, 1. J. Rodriguez, Ihys. Rev. Ag. 9(0) (1973).

17] 'T. R. Sosnick, W'. M. Snow, P'. I:. Sokol, R. N. Silver, this volume.

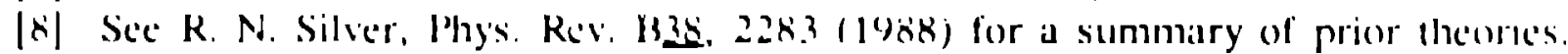

(9) 1. J. Rodgriguez, 11. A. Cersch, 11. A. Mook, Phys ?3e. A2. 2085 (19)7.1.

[10) R. N. Silver, (i. Reiter, Rap. Comem. I'hys. Rev. 131 35, 3647 (1987).

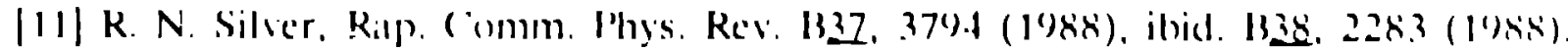

|12| T. R Sosnick. W'. M. Snow, I'. I:. S(uh:), R. N. Silver, lo be published.

|1.3| T. R. Sosnich, W: M. Sinow, I' I:. Suke,l, this volume.

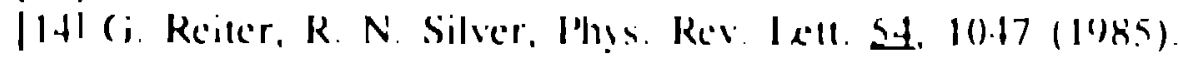

$11.5 \mid$; Reiter, this volumic.

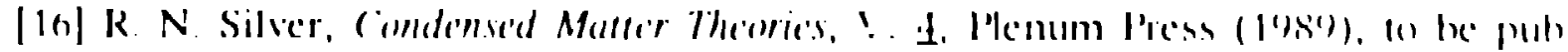
lished.

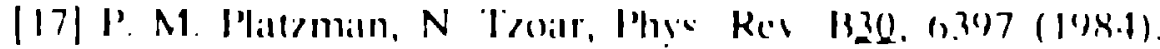

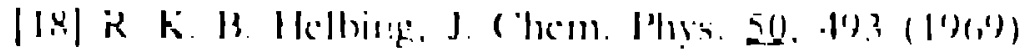

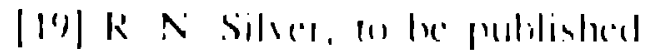

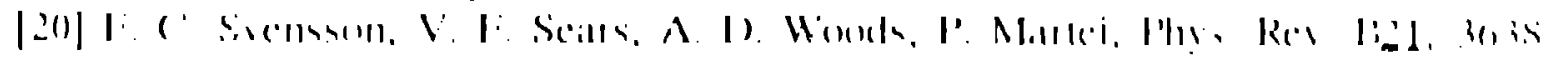
(I)

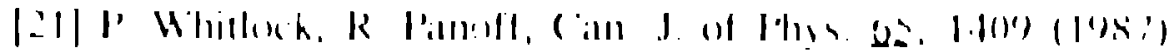

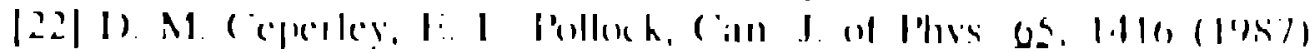

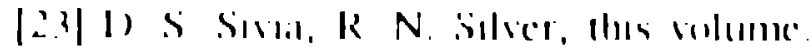

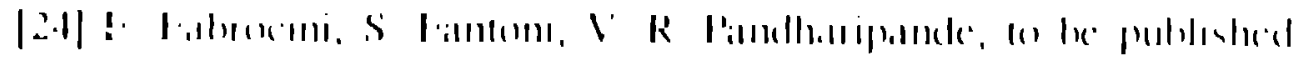

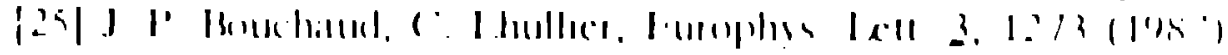

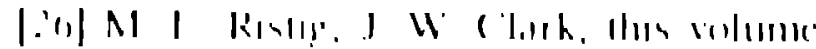

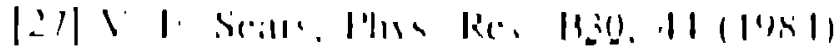

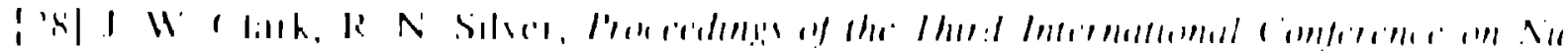

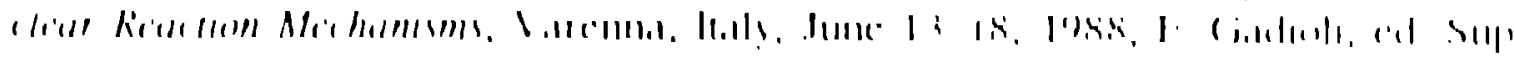

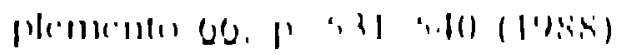

